Brücken, Wasserleitungen und Schulhäuser. Es drängt sich die Frage auf: Warum ist Nepal heute das wohl am meisten «unterentwickelte» Land der Erde, obschon die Naturgrundlagen ähnlich sind wie in der Schweiz, und die handwerklichen Fähigkeiten der Nepali (wofür die prachtvollen Häuser und Tempel z. B. der Newari sprechen) sowie die menschlichen Qualitäten (wofür die weltberühmten Gurkhasoldaten mit ihrem Fleiß, ihrer Ehrlichkeit und Zuverlässigkeit sowie mit ihrem Mut Zeugnis ablegen) dem Schweizervolk in nichts nachstehen. Jeder Nepalkenner wundert sich immer wieder, daß die Nation Nepals überhaupt im heutigen Umfang besteht, wo doch kaum ein gemeinschaftlicher Geist vorhanden ist, abgesehen von einem (sich oft nachteilig auswirkenden) unbändigen Freiheitswillen. Die heterogene $\mathrm{Zu}$ sammensetzung des nepalischen Volkes, vor allem aber die außergewöhnliche regionale Verteilung der einzelnen Volksgruppen mag eine plausible Antwort geben: In Tälern, in welchen sich so grundsätzlich verschiedene Völker auf engem Raum je nach Höhenlage in die Besiedlung teilen, konnte gar kein Gemeinschaftsgefühl entstehen. Man findet denn auch Ansätze für Gemeinwesen bis jetzt nur in Gebieten, wo die Bevölkerung einheitlich ist, wie z. B. in den Newarstädten, oder in den hochgelegenen Tälern mit homogener Sherpa- oder Tibeter-Bevölkerung.

\title{
WICHTIGSTE LITERATUR
}

Fürer-Haimendorf, Ch. von (1954). Bei Bauernstämmen Ostnepals. Neue Zürcher Zeitung 19. 9. 1954. - Derselbe (1954). Besuch im Lande der Sherpas. Neue Zürcher Zeitung 17. 10. 1954. Heuberger, H. (1956). Der Weg zum Tscho Oyu. Mitt. d. Geograph. Ges. Wien, Bd. 98, Heft I. -Lobsiger-Dellenbach M. (1952). Himalaya du Népal. Mission scientifique Genevoise. Editions Jeheber, Genève - Paris. - Rauch, E.: Eindrücke eines Fluges über Nepal. Geographica Helvetica VIII, 1953, Nr. 4. - Derselbe: L’agriculture du Népal. Le Globe, 96, 1955.

\section{THE REGIONS AND POPULATIONS OF NEPAL}

The population of Nepal consists of at least 15 different groups. There are three main groups : the native Tibeto-Birman group of the Midlands, the Tibetan group of the Tibetan plateau and in the valleys in the main range of the Himalayas, and the Indian group, which has intruded from India. The Tibeto-Birman group consists of the Newars, Gurungs, Thamangs, Rais, Limbus, Thakuris, Mangars, Rukhas and Buras. The Sherpas and the pure Tibetans represent the Tibetan group, while the orthodox hinduistic group is composed of the highest casts only, the Brahmins and the Kschatrias (priests and warriors). All the above mentioned people have their own language (excepted Brahmins and Kschatrias), originally their own religion; further their own customs, celebrations, types of houses and settlements. Outstanding feature of the different people is their extraordinary distribution according to altitudes: The hinduistic group is living in the valleys, not higher than $2000 \mathrm{~m}$, the Tibeto-Birman group between $1500 \mathrm{~m}$ and $2400 \mathrm{~m}$ above sea level, while the Sherpas and the Tibetans are found above appr. $2200 \mathrm{~m}$.

\section{CARTE DE L'ÉCONOMIE MONDIALE}

\section{$1: 32000000$}

\section{Hans Boesch}

\section{QUELQUES REMARQUES ESSENTIELLES}

a) La carte de l'économie mondiale a été élaborée tout spécialement à l'usage des écoles secondaires et des gymnases. C'est la raison pour laquelle nous avons prié de nombreux professeurs de nous donner leur avis sur des cartes déjà existantes et de nous soumettre des suggestions pour une nouvelle carte. Il en est résulté une série de principes didactiques, dont il a fallu tenir compte. La carte à l'usage de l'enseignement doit avant tout donner des réponses claires et nettes à l'élève qui y cherche la solution des problèmes que lui pose le professeur. Une carte, si belle soit-elle, mais qui ne suffit

Editeur: Kümmerly \& FreY, Editions géographiques, Berne

Rédaction: Institut de Géographie de l'Université de Zurich, H. Boesch, Professeur, et A. Behrens, M. Muller, F. Casatello 
pas à ces exigences, ne convient pas à l'enseignement. Les questions peuvent tout aussi bien se référer à des détails qu'à un ensemble. Il faut donc pouvoir reconnaître facilement chaque signe et en même temps discerner un groupe de détails formant un tout. Bien que ces principes didactiques aient été le point de départ de tout ce qui suit, la présente carte de l'économie mondiale se prête également comme carte d'ensemble pour des bureaux, etc., car elle donne des renseignements précis sur l'importance d'une région dans l'ensemble de l'économie mondiale.

b) Les principes scientifiques sont discutés dans les paragraphes ci-dessous. Ces principes ont déterminé le contenu de la carte, le choix des signes et le degré de la généralisation. Le caractère scientifique de la carte exige que les données soient correctes et puissent être contrôlées à l'aide des sources d'information mentionnées. Les sources statistiques les plus importantes sont les annuaires des Nations Unies et de leurs organisations.

c) Principes cartographiques. La représentation de l'utilisation du sol constitue le fond de la carte sur lequel figurent les points détaillés qui sont indiqués au moyen de symboles. L'intensité des couleurs de fond a été nuancée selon l'importance qu'il faut attribuer au sujet représenté; cependant les couleurs sont moins fortes que celles des symboles qui s'y détachent nettement. L'intensité de ces derniers a été choisie de telle sorte que les centres de l'économie mondiale puissent être discernés au premier coup d'œil. Les symboles ont été conçus aussi conformes que possible à la réalité et apparaissent à l'emplacement qui leur revient dans chaque pays. Tout schématisme a été évité ; par exemple, les signes n'ont pas été disposés en ligne. De ce fait, l'aspect de la carte est rendu plus vivant et plus réel.

La projection de la carte (Van der Grinten) ne satisfait pas entièrement, les superficies ne correspondant pas à la réalité. Cela se remarquera surtout par les agrandissements considérables dans l'hémisphère nord (Canada, U.R.S.S.). Nous avons néanmoins choisi cette projection, faute de mieux, et parce qu'elle présente plusieurs avantages au point de vue de l'enseignement. Pour cette raison son usage est en général très répandu dans les écoles. A quelques exceptions près, nous avons omis de faire des inscriptions sur cette carte, afin de ne pas surcharger inutilement son aspect. Comme il existe une carte politique mondiale (Kümmerly \& Frey, 1:32000 000) de même projection et présentation graphique, pourvue d'une riche nomenclature et pouvant être employée en même temps que la carte de l'économie mondiale, ce défaut est compensé par une plus grande clarté de l'image.

\section{UTILISATION DU SOL}

a) Les documents, permettant de présenter par des moyens cartographiques l'utilisation du sol sur toute la surface de la terre, sont, pour le moment, très précaires. Il n'existe jusqu'à présent que quelques pays (Grande Bretagne; Etats-Unis, Japon, etc. partiellement) qui aient exécuté des cartes à grande échelle de l'utilisation du sol. Les principes établis par l'Union Géographique Internationale ont apporté une certaine unité quant à la différentiation des types de l'utilisation du sol et ont amené de grands progrès dans l'exécution des cartes de ce genre. Cependant, nous sommes encore loin de pouvoir élaborer une carte se basant uniquement sur des levés originaux. Pour la carte de l'économie mondiale, nous avons employé plusieurs documents avec des légendes en partie différentes qu'il a fallu d'abord faire concorder. Il s'agit avant tout de E. LEHMANN: «Weltatlas», Leipzig 1952, «Oxford Economic Atlas of the World», 1955 et «Oxford Regional Economie Atlas: The U.S.S. R. and Eastern Europe», 1956. En outre, nous avons utilisé une carte de l'U. S. Department of Agriculture, Office of Foreign Agricultural Relations, qui montre l'étendue des terres arables et, dans quelques cas, également le «Chamber of Commerce Atlas», London, datant de 1925. 
Les statistiques concernant l'utilisation agraire donnent par contre de plus amples détails; on peut à ce sujet se référer aux données concernant les différents pays publiées par l'«Annuaire de statistiques agricoles et alimentaires» de la F.A.O. Toutefois pour la rédaction cartographique, ces valeurs sont en général inutilisables; elles ne servent qu'à corriger le tableau de la carte déjà obtenu dans les cas où une forte division politique existe. Les statistiques de la F. A. O. différencient comme suit les types de l'utilisation du sol et les superficies de tout le globe:

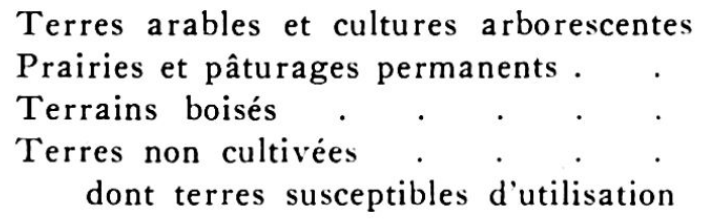

1322000000 ha
2363000000 ha
$39+7000000$ ha
5859000000 ha
+31000000 ha

b) Les différents types suivants de l'utilisation du sol apparaissent sur la présente carte:

$1^{\circ}$ Terres incultes: Correspond à «terres non cultivées» y compris une partie des «terres susceptibles d'utilisation» d'après les statistiques de la F.A.O.; de même les régions polaires bien qu'elles ne soient pas mentionnées dans les statistiques de la F.A.O. Paysage naturel.

$2^{\circ}$ Forêts: «Terrains boisés selon statistiques de la F. A. O. Y figurent seulement, conformément au degré de généralisation, les vastes superficies recouvertes de forêts. En général paysage naturel. Parfois certaine influence culturelle.

$3^{\circ}$ Pâturages et chasse: Comprend «prairies et pâturages permanents 》et partiellement «terres susceptibles d'utilisation» (surtout dans latitudes tempérées et tropicales, les régions avec possibilité d'irrigation) et partie des «terres non cultivées», celles des régions subpolaires, utilisées pour la chasse. La culture n'a presque pas transformé le paysage, en majeure partie paysage naturel.

$4^{\circ} \mathrm{Cultures}:$ Correspond à «terres arables et cultures arborescentes》selon statistiques de la F.A.O. Transformation intense du paysage par l'exploitation agraire. 'Technique agricole, plantes de cultures, organisation économique très variées. Paysage culturel.

La division en 4 types (1-4) de l'utilisation du sol correspond à l'intensification progressive de l'influence humaine sur le paysage et de ce fait au développement graduel du paysage naturel en paysage culturel (dans le cas spécial: en paysage agraire). Un aperçu historique montrerait les grandes superficies qui, au cours du XIXème siècle ont été transformées en paysage culturel. D'autre part, en consultant les statistiques de la F.A.O., on s'aperçoit que les possibilités d'expansion des superficies agricoles sont minimes. Cela, tenant compte de l'accroissement considérable de la population sur toute la terre, nous amène à un problème essentiel et vital du XXème siècle, celui du rapport entre la production agricole et les besoins de la population.

$\begin{aligned} \text { Population mondiale } \quad . \quad 1920: & 1,810 \text { millions } \\ 1930: & 2,013 》 \\ 1940: & 2,250 》 \\ 1950: & 2,504 》 \\ 1954: & 2,652 》\end{aligned}$

$5^{\circ}$ Pêche commerciale: Seules les régions de pêche importantes pour le commerce de gros y figurent.

\section{VILLES ET INDUSTRIES}

a) Les centres de gravité, sur lesquels s'est concentrée de plus en plus l'économie mondiale, ont été créés par le procès d'industrialisation et d'urbanisation au cours des XIXème et XXème siècles. Ces centres doivent donc ressortir tout spécialement sur la carte. Des signes d'un rouge vif ont été employés pour représenter les villes et les 
industries, tandis que pour l'exploitation minière et l'industrie extractive, on a choisi le noir, couleur moins voyante, conformément à l'influence moins considérable de ces dernières sur la formation de centres de gravité. Pouvant constater un accroissement constant de la population, de la production industrielle et minière pendant les dernières années, nous avons préféré utiliser les dernières données statistiques, en général celles de 1953, plutôt que de calculer une valeur moyenne basée sur des valeurs de plusieurs années consécutives.

L'élaboration de la carte a été rendue difficile par le manque de statistiques soviétiques. Comme les sources d'information concernant l'U. R. S. S. ne nous indiquent que des valeurs approximatives, nous avons toujours pris comme total mondial le total de tous les pays sauf celui de l'Union soviétique et avons ajouté à ce total les valeurs russes compilées. A cet effet, le «Minerals Yearbook» de l'U. S. Department of Commerce a servi de source importante.

b) Villes. Déjà par le nombre de leurs habitants, les grandes villes forment des centres importants de la vie économique. C'est pourquoi toutes les villes de plus d'un million d'habitants ont été marquées sur la carte par un symbole circulaire de couleur rouge. Toutefois, le nombre d'habitants d'une ville varie selon qu'on se réfère à l'unité politique ou à toute l'agglomération. Ainsi nous obtenons pour New York, la région urbaine la plus grande du monde, des valeurs fort différentes: New York, unité politique, 7,891,957 habitants, agglomération de New York $12,296,117$ habitants. Au point de vue économique, le nombre d'habitants de toute l'agglomération est important. Les statistiques qui étaient à notre disposition, par contre, se réfèrent en général à l'unité politique d'une ville (par exemple le «Statesman's Yearbook» ou le «Webster's Geographic Dictionnary »).

$1^{\circ}$ Villes de plus de 1,000,000 d'habitants: Le nombre d'habitants se réfère à l'unité politique. Voir table I.

$2^{\circ}$ Villes industrielles: Villes de plus de 200,000 habitants avec industries importantes. Choix selon «Staatesman's Yearbook», «Webster's Geographic Dictionnary » et publications spéciales. Voir table II.

c) Industrie. La représentation de l'industrialisation d'un pays, se basant sur les statistiques, présente de grosses difficultés, car la définition des termes «industrie» et «ouvrier industriel»varient d'un pays à l'autre. Il a donc fallu trouver d'autres moyens pour faire ressortir sur la carte la formation d'un centre de gravité. En premier lieu, nous avons mis en valeur la production de l'acier brut qui, pour la puissance industrielle d'un pays, est considérée comme décisive. L'importance de ces régions est mise en relief par l'inscription des villes industrielles. En outre, les symboles, représentant les exportations industrielles importantes, montrent les pays industriels qui, pour le commerce international, entrent tout spécialement en ligne de compte. La forte densité des signes correspond à la concentration de l'industrie dans la réalité. Cette accumulation nécessite un choix minutieux de ce qu'il faut représenter et omettre sur la carte.

$1^{\circ}$ Acier brut: Production mondiale (sans U.R.S.S.) 195 millions de t.; Union soviétique 38 millions de t. Détermine les centres de l'industrie lourde. Valeurs de l'acier brut toujours plus élevées que celle de la fonte (139, resp. 26 millions de t.).

2o Exportation de produits industriels: Valeur de l'unité 1 milliard de $\$$. Une valeur d'unité équivalente à celle des symboles agricoles (25 millions de \$) n'a pas pu être réalisée pour des raisons graphiques. Nous avons remédié à ce défaut en accentuant davantage la forme et la couleur du signe.

d) Exploitation minière. La représentation de l'exploitation minière a posé moins de problèmes que celle de l'industrie. Les annuaires des Nations Unies et le "Minerals Yearbook» de l'U.S. Department of Commerce contiennent toutes les données néces- 
saires; toutefois, celles concernant l'Union soviétique ne sont souvent que des valeurs évaluées (voir remarques préliminaires). En général, il a été facile de placer correctement les signes de l'exploitation minière grâce à d'excellents travaux analythiques et régionaux. Les produits miniers sont classés en deux catégories. La première comprend le minerai de fer, la houille et le pétrole brut. Elle présente non seulement une extraction très élevée, mais influence en plus d'une manière décisive le développement industriel. Dans la deuxième figurent tous les autres produits miniers qu'on peut qualifier de secondaires, les quantités extraites étant moins considérables et le rôle de leurs industries moins important que dans la première catégorie.

$1^{\circ}$ Minerai de fer: Total mondial du minerai de fer extrait converti en fer contenu. 125 millions de t. (sans U.R.S. S.) ; U.R.S. S. 30 millions de t. Le fer contenu varie généralement entre 30 et $60 \%$ du minerai de fer. La concentration sur quelques centres nous a fait choisir des symboles en forme de filet; chaque nœud représente $1 \%$ du total mondial.

$2^{\circ} \mathrm{Hou}$ ille: Comprend toutes les sortes de charbon, elles sont calculées en unités de houille bitumineuse. Total mondial de l'extraction de houille 1,200 millions de t. (sans U. R. S. S.), U. R. S. S. 320 millions de t.

3o Pétrole brut: Total mondial de l'extraction de pétrole brut 605 millions de t. (sans U. R.S.S.), U.R.S.S. 52 millions de t. Les gaz naturels n'ont pas été pris en considération faute de données statistiques complètes. Pour la même raison l'industrie pétrochimique sur la base pétrole-gaz naturels n'a pas été inscrite sur la carte. Les raffineries, qui ces dernières années ont été construits de préférence dans les régions de consommation, ont du être omises pour des raisons graphiques (manque de place).

$4^{\circ}$ Produits miniers secondaires: Les symboles chimiques sont employés au lieu de signes. Pour éliminer toutes les régions minières de peu d'importance, nous n'avons tenu compte que de celles ayant au moins une part de $5 \%$ du total mondial. Deux catégories (5-25\%, plus de $25 \%$ ). Pour de plus amples détails voir table III.

\section{AGRICULTURE ET ÉCONOMIE FORESTIÈRE}

a) Remarques préliminaires. Les quantités de ce groupe faisant l'objet du commerce mondial sont représentées par leurs valeurs d'exportation et non par les valeurs de production. Cette représentation ne se rencontre guère sur des cartes économiques; nous l'avons toutefois choisie pour différentes raisons dont voici les quatre les plus importantes. Premièrement, comparées avec les statistiques minières, celles des produits agricoles sont incomplètes. Pour de nombreux produits, n'ayant de l'importance que pour l'économie indigène (par exemple le millet), les données sont nettement insuffisantes. Une carte de production de tous les produits agraires mentionnés dans les statistiques se révélerait donc pleine de lacunes, et, dans de nombreuses régions, l'agriculture ne serait pas du tout représentée par des signes. Deuxièmement, la couleur de fond relative aux «cultures» nous renseigne où, sous les formes diverses de l'agriculture, la terre est cultivée. Ainsi, en comparant le fond avec les signes d'exportation, on peut discerner les régions dans lesquelles l'agriculture ne produit que pour le marché local et celles où elle est orientée vers l'exportation. Troisièmement, si l'on se demande par exemple quelles sont les régions importantes pour la production des bananes, on aimerait connaître généralement les grands fournisseurs de l'économie mondiale (Amérique centrale, les îles Canaries) et non pas toute contrée où l'on cultive le bananier en abondance (par exemple Afrique orientale, consommation locale). Quatrièmement, grâce à cette méthode, les régions industrielles se trouvent fortement déchargées quant à la densité des signes; dans ces régions la terre est en général cultivée d'une manière intense, et les produits sont consommés sur place. 
b) La détermination de la valeur des signes de ce groupe a causé quelques difficultés. Nous avons essayé tout d'abord de concevoir des signes ressemblant si possible au sujet représenté par la forme et les couleurs et avons en outre attribué les mêmes couleurs aux produits apparentés. En principe, chaque produit est symbolisé par un signe, et chaque signe a une valeur spécifique. Comme les statistiques d'exportation se réfèrent aux quantités des produits (t., hl., etc.), cette valeur spécifique doit être exprimée dans les mêmes unités. D'autre part, les signes doivent correspondre entre eux dans leur valeur monétaire afin qu'ils aient tous une signification économique équivalente. C'est pourquoi nous avons multiplié les différents totaux d'exportation avec le ou les prix mondiaux des produits en question et avons ainsi obtenu une valeur commune (valeur monétaire). En attribuant à chaque signe agricole une valeur d'exportation de $25000000 \$$, on arrive à un nombre global raisonnable de signes qui ne surcharge pas l'aspect de la carte. Ce nombre s'élève à plus de 500 . Les pommes de terre, le colza, etc. ne sont représentés que par un seul signe, tandis que le froment en accuse le plus grand nombre (87). C'est au moyen de cette méthode que les valeurs pondérées, figurant sur la légende de la carte, ont été déterminées. Par la densité des signes, l'importance économique d'une région peut donc être reconnue au premier coup d'œil, et la comparaison entre les différents pays est facile à effectuer.

En prenant en considération la diversité et le grand nombre des symboles, il a fallu également faire concorder leur valeur d'intensité. Celle-ci est déterminée par les couleurs, la grandeur et la forme et par l'entourage du signe sur la carte. Par exemple, le jaune vif du beurre et du fromage a été compensé par une dimension restreinte du symbole; par contre le signe vert du bois, toujours placé sur un fond de même couleur, est un peu plus grand que les autres.

Ce choix des valeurs d'exportation a le désavantage de n'attribuer que peu de signes à de grands pays comme l'U.R.S.S. à relations commerciales restreintes. Il serait évidemment faux de conclure que ces pays ne présentent qu'une production agraire insignifiante. La division politique plus poussée en Europe qu'en Amérique du Nord se fait remarquer par une augmentation de signes pour les pays de notre continent (par exemple, aux Pays-Bas, au Danemark, etc.). Toutefois, en étudiant l'économie internationale, il est essentiel de faire ressortir ces différences caractéristiques. Sur une carte où la production seule est représentée, on ne saurait jamais les retrouver.

Contrairement à la production minière, nous n'avons pas employé les dernières données statistiques à notre disposition, sinon calculé une valeur moyenne basée sur celles des années 1951-1953.

Les signes apparaissent à l'emplacement qui leur revient dans chaque pays. Si, pour un pays à plusieurs régions productrices, nous n'avions qu'un seul signe à disposition, nous l'avons placé dans la région la plus importante et non pas dans un centre déterminé mathématiquement. Pour le choix de cet emplacement, la part de la production destinée à l'exportation a été plus déterminante que la quantité produite.

c) Les différents produits. Pour de plus amples détails voir table IV.

\section{LE COMMERCE MONDIAL}

Nous avons omis de représenter sur la présente carte les rapports économiques en forme de bandes symbolisant le transport et le commerce. Cela peut étonner et doit donc être motivé.

Il aurait fallu présenter graphiquement le transport commercial (avant tout le trafic maritime) et le commerce international. Dans les deux cas les données statistiques auxquelles on aurait pu se référer se sont révélées insuffisantes. Les statistiques de transport (maritime) ne se réfèrent qu'au trafic des lignes régulières et laissent ainsi de côté la plus grande partie du trafic maritime des marchandises, spécialement 
important pour nous. Les statistiques d'exportation, d'autre part, sont complètes, mais nous ne savons rien quant à la route que prennent ces exportations. Il serait donc possible de faire un diagramme représentant les relations du commerce international, tandis que la compilation d'une carte poserait des problèmes considérables.

Ce sont ces raisons pour lesquelles nous avons préféré ne représenter sur la carte ni le transport ni le commerce international.

Au cours des préparatifs pour l'élaboration de la carte, nous avons été surpris d'entendre de nombreux professeurs se prononcer contre la présentation de pareilles bandes vu que l'interprétation de la carte en serait rendue plus difficile.

D'autre part, le fait que les relations économiques internationales sont soumises à un changement continuel - surtout dans la période d'après-guerre $\leftarrow$ n'a pas influencé notre décision, sinon nous n'aurions pu attribuer des valeurs d'exportation aux produits agraires. Une carte de l'économie mondiale est un document temporaire qui doit constamment être réadapté aux circonstances du moment.

Table I

Tabelle I

Villes de plus de 1000000 d'habitants

Städte mit mehr als 1000000 Einwohnern

\begin{tabular}{|c|c|c|c|c|c|}
\hline 1. London & 8,3 Mio & 22. São Paulo & 2,o Mio & 43. Manila & 1,2 Mio \\
\hline 2. New York & 7,9 & 23. Osaka & 1,9 & +t. Wuhan & 1,2 \\
\hline 3. Tokyo & 6,3 & 24. Detroit & 1,8 & 45. Birmingham & 1,1 \\
\hline 4. Shanghai & 6,2 & 25. Sydney & 1,8 & 46. Hyderabad & 1,1 \\
\hline 5. Moskwa & 4,1 & 26. Hamburg & 1,7 & 47. Kyoto & $.1,1$ \\
\hline 6. Chicago & 3,6 & 27. Roma & 1,7 & 48. Singapore & 1,1 \\
\hline 7. Berlin & 3,5 & 28. Budapest & 1,6 & 49. Bangkok & 1,0 \\
\hline 8. Leningrad & 3,1 & 29. Madrid & 1,6 & 5o. Bucuresti & 1,0 \\
\hline 9. Buenos Aires & 3,0 & 3o. Saigon & 1,6 & 51. Glasgow & 1,0 \\
\hline 1o. Bombay & 2,8 & 31. Chungking & 1,6 & 52. Istanbul & 1,0 \\
\hline 11. Paris & 2,8 & 32. Wien & 1,6 & 53. Karachi & 1,0 \\
\hline 12. Peking & 2,8 & 33. Canton & 1,5 & 54. København & 1,0 \\
\hline 13. Tientsin & 2,7 & 34. Shenyang & 1,5 & 55. Montreal & 1,0 \\
\hline 14. Calcutta & 2,5 & 35. Madras & 1,4 & 56. Nagoya & 1,0 \\
\hline 15. Djakarta & 2,5 & 36. Melbourne & 1,4 & 57. Nanking & 1,0 \\
\hline 16. Rio de Janciro & 2,3 & 37. Sœul & 1,4 & 58. Napoli & 1,0 \\
\hline 17. Hongkong & 2,2 & 38. Athinai & 1,3 & 59. Port Arthur- & \\
\hline 18. Mexico & 2,2 & 39. Barcelona & 1,3 & Dairen & 1,0 \\
\hline 19. Cairo & 2,1 & 4o. Santiago & 1,3 & 6o. Praha & 1,0 \\
\hline 2o. Philadelphia & 2,1 & 41. Delhi & 1,2 & 61. Warszawa & 1,0 \\
\hline 21. Los Angeles & 2,0 & 42. Milano & 1,2 & 62. Yokohama & 1,0 \\
\hline
\end{tabular}

Table II

Villes industrielles de plus de 200000 habitants

Tabelle II Industriestädte über 200000 Einwohner

\section{AMÉRIQUE DU NORD - NORDAMERIKA}

Hamilton, Ottawa, Toronto, Vancouver. Akron, Atlanta, Baltimore, Birmingham, Boston, Buffalo, Cincinnati, Cleveland, Columbus, Dallas, Dayton, Denver, Fort Worth, Houston, JerseyCity, Kansas-City, Long Beach, Louisville, Memphis, Milwaukee, Newark, Norfolk, Oakland, Oklahoma, Omaha, Pittsburgh, Portland, Providence, Rochester, Salt Lake City, San Antonio, San Diego, San Francisco, Seattle, St. Louis, St. Paul, Syracuse, Tampa, Toledo, Tulsa, Washington, Wichita, Worcester. Guadalajara, Monterrey, Puebla. Havanna. San Juan.

\section{AMÉRIQUE DU SUD - SÜDAMERIKA}

Caracas, Maracaibo. Lima. Bogotá, Cali, Medellin. La Paz. Porto Alegre, Recife. Rosario. Montevideo. 


\section{EUROPE - EUROPA}

Oslo. Göteborg, Stockholm. Helsinki. Dublin, Bradford, Bristol, Cardiff, Coventry, Croydon, Edinburgh, Hull, Leeds, Leicester, Liverpool, Manchester, Northampton, Nottingham, Plymouth, Portsmouth, Sheffield, Stoke on Trent. Lisboa, Porto. Bilbao, Malaga, Valencia, Zaragoza. Bordeaux, Lyon, Marseille, Nantes, Nice, Toulouse. Antwerpen (Anvers), Bruxelles. Amsterdam, Rotterdam. Bochum, Braunschweig, Bremen, Chemnitz, Dortmund, Dresden, Duisburg, Düsseldorf, Essen, Frankfurt a/M., Gelsenkirchen, Halle, Hannover, Kiel, Köln, Leipzig, Lübeck, Magdeburg, Mannheim, München, Nürnberg, Oberhausen, Stuttgart, Wiesbaden, Wuppertal. Basel, Zürich. Bari, Bologna, Catania, Firenze, Genova, Messina, Palermo, Torino, Venezia. Graz. Brno. Wroclaw, Krakow, Lodz, Poznan, Szczecin. Beograd, Zagreb. Sofia.

\section{U.R.S.S. - SOWJETUNION}

Baku, Chelyabinsk, Chita, Dnepropetrovsk, Gor'kiy, Irkutsk, Karaganda, Kazan', Kemerovo, Khabarovsk, Khar'kov, Kiev, Krasnoyarsk, Kuybyshev, L'vov, Magnitogorsk, Minsk, Molotov, Nizhniy Tagil, Novosibirsk, Odessa, Omsk, Riga, Rostov, Saratov, Stalingrad, Stalino, Stalinsk, Sverdlovsk, Tashkent, Tbilisi, Tula, Ufa-Chernikovsk, Vladivostok, Voronezh, Yaroslavl', Yerevan, Zaporozh'ye.

\section{V. $\triangle S I E-\triangle S I E N$}

Haifa, Tel Aviv. Ahmedabad, Amritsar, Baroda, Howrah, Jamshedpur, Kanpur, Lucknow, Madura, Nagpur, Poona, Surat. Colombo. Lahore, Rawalpindi. Hangchow. Anshan, Fushun. Tai-peh. Amagasaki, Fukuoka, Gifu, Himeji, Kagoshima, Kanazawa, Kawasaki, Kobe, Kokura, Nagasaki, Niigate, Sakai, Sapporo, Sendai, Shizuoka, Yahata.

$$
\text { IV. AFRIQUE - AFRIKA }
$$

Durban, Johannesburg, Capetown, Port Elizabeth, Pretoria.

$$
\text { VII. AUSTRALIE et OCÉANIE - AUSTRALIEN und OZEANIEN }
$$

Adelaide, Brisbane.

\begin{tabular}{|c|c|c|c|}
\hline Produit & Produkt & $\begin{array}{c}\text { Production mondiale, } \\
\text { sans U.R.S.S. } \\
\text { Weltproduktion, ohne U.S.S.R. } \\
(1953)\end{array}$ & $\begin{array}{l}\text { U.R.S.S. } \\
\text { U.S.S.R. } \\
\text { approx. } \\
\text { (1952) }\end{array}$ \\
\hline Bauxite & Bauxit & $12500000 \mathrm{t}$ & $750000 \mathrm{t}$ \\
\hline Plomb & Blei & $1720000 \mathrm{t}$ & . \\
\hline Chrome & Chrom & $1400000 t$ & too $000 \mathrm{t}$ \\
\hline Diamants & Diamanten & $\begin{array}{c}20100000 \text { carats métriques } \\
\text { Meter-Karat }\end{array}$ & . \\
\hline Or & Gold & $75+\mathrm{t}$ & $300 t$ \\
\hline Potasse & Kali & $5400000 t$ & . \\
\hline Cuivre & Kupfer & $2440000 t$ & $300000 \mathrm{t}$ \\
\hline Manganèse & Mangan & 2800000 & $1000000 t$ \\
\hline Molybdène & Molybdän & $28520 \mathrm{t}$ & . \\
\hline Nickel & Nickel & $175000 \mathrm{t}$ & $25000 \mathrm{t}$ \\
\hline Nitrate & Nitrat & $20000000 \mathrm{t}$ & . \\
\hline Phosphates & Phosphat & $22700000 \mathrm{t}$ & $3000000 t$ \\
\hline Pyrites & Pyrit & $11800000 t$ & . \\
\hline Mercure & Quecksilber & $4850 \mathrm{t}$ & too $t$ \\
\hline Soufre & Schwefel & $5800000 \mathrm{t}$ & . \\
\hline Argent & Silber & $5900 \mathrm{t}$ & $800 \mathrm{t}$ \\
\hline Vanadium & Vanadium & $5070 \mathrm{t}$ & . \\
\hline $\begin{array}{l}\text { Wolfram } \\
\text { (Tungstène) }\end{array}$ & Wolfram & $39000 t$ & $7500 \mathrm{t}$ \\
\hline Zinc & Zink & $2500000 t$ & $200000 t$ \\
\hline Etain & Zinn & $178800 \mathrm{t}$ & . \\
\hline
\end{tabular}




\begin{tabular}{|c|c|c|c|}
\hline Produit & Produkt & $\begin{array}{c}\text { Total du commerce } \\
\text { mondial sans U.R.S.S. } \\
\text { Weltexportmenge } \\
\text { ohne U.S.S.R. } \\
\text { Moyenne - Mittel } \\
1951 \text { - } 1953\end{array}$ & $\begin{array}{l}\text { Principaux pays exportateurs* } \\
\text { Wichtigste Exportländer* }\end{array}$ \\
\hline Bois & Holz & $1618560000 \mathrm{US} S$ & $\begin{array}{l}\text { Canada (16), Suède-Schweden (9), } \\
\text { Finlande-Finnland (8), Autriche- } \\
\text { Österreich (4), E. U.-U.S.A. (4) }\end{array}$ \\
\hline Espèce bovine & Rinder & $\begin{array}{l}1766 \text { ooo } \\
\text { Têtes-Köpfe }\end{array}$ & $\begin{array}{l}\text { Irlande-Irland (5), Danemark-Dä- } \\
\text { nemark (2) }\end{array}$ \\
\hline Viande de bœuf & Rindfleisch & $773000 \mathrm{t}$ & $\begin{array}{l}\text { Australie-Australien (4) } \\
\text { Argentine-Argentinien ( } 3 \text { ) }\end{array}$ \\
\hline Viande de porc & Schweinefleisch & $411600 \mathrm{t}$ & $\begin{array}{l}\text { Danemark-Dänemark (6), Pologne- } \\
\text { Polen (2) }\end{array}$ \\
\hline Beurre & Butter & $4+0000 \mathrm{t}$ & $\begin{array}{l}\text { Nouvelle-Zélande-Neuseeland (10) } \\
\text { Danemark-Dänemark (8), Pays- } \\
\text { Bas-Niederlande (3) }\end{array}$ \\
\hline Fromage & Käse & $358300 \mathrm{t}$ & $\begin{array}{l}\text { Nouvelle-Zélande-Neuseeland (4) } \\
\text { Pays-Bas-Niederlande (3), Dane- } \\
\text { mark-Dänemark (2) }\end{array}$ \\
\hline Pommes de terre & Kartoffeln & $1866600 \mathrm{t}$ & Pays-Bas-Niederlande (1) \\
\hline $\mathrm{Riz}$ & Reis & $4850000 t$ & $\begin{array}{l}\text { Thaïlande - Thailand (7), Birma- } \\
\text { nie-Burma (6), E. U.-U.S. A. (3) }\end{array}$ \\
\hline $\begin{array}{l}\text { Froment et } \\
\text { farine de froment }\end{array}$ & $\begin{array}{l}\text { Weizen und } \\
\text { theizenmehl }\end{array}$ & $27550000 \mathrm{t}$ & $\begin{array}{l}\text { E. U.- U.S.A. (35), Canada (31), } \\
\text { Australie-Australien (9) }\end{array}$ \\
\hline Orge & Gerste & $5183300 \mathrm{t}$ & Canada (t), E.U.-U.S.A. (2) \\
\hline Seigle & Roggen & $933300 \mathrm{t}$ & $\begin{array}{l}\text { Canada (1), Argentine - Argenti- } \\
\text { nien (1) }\end{array}$ \\
\hline Avoine & Hafer & $1793300 \mathrm{t}$ & Canada (3) \\
\hline Maïs & Mais & $4750000 t$ & $\begin{array}{l}\text { E. U.- U.S. A. (7), Argentine-Ar- } \\
\text { gentinien (2) }\end{array}$ \\
\hline Café & Kaffee & $1990000 \mathrm{t}$ & $\begin{array}{l}\text { Brésil-Brasilien ( } 38) \\
\text { Colombie-Columbien }\end{array}$ \\
\hline Cacao & Kakao & $708300 \mathrm{t}$ & Ghana (9), Nigeria-Nigerien (5) \\
\hline Thé & Tee & $468300 \mathrm{t}$ & $\begin{array}{l}\text { Inde-Indien (8), Ceylan-Ceylon } \\
(6)\end{array}$ \\
\hline $\begin{array}{l}\text { Sucre brut } \\
+ \text { raffiné }\end{array}$ & $\begin{array}{l}\text { Zucker, roh } \\
+ \text { raffiniert }\end{array}$ & $13816600 \mathrm{t}$ & $\begin{array}{l}\text { Cuba ( } 8) \text {, Porto-Rico-Puerto-Rico } \\
\text { (2), Hawaii (2) }\end{array}$ \\
\hline Tabac & Tabak & $593300 \mathrm{t}$ & $\begin{array}{l}\text { E.U.-U.S.A. (11), Turquie-Tür- } \\
\text { kei (3) }\end{array}$ \\
\hline Vin & Wein & 17 too ooo hl & Algérie-Algerien (6) \\
\hline Agrumes & Citrusfrüchte & $2466600 t$ & $\begin{array}{l}\text { Espagne-Spanien (5), E. U.-U.S.A. } \\
\text { (2) }\end{array}$ \\
\hline Bananes & Bananen & $2566600 \mathrm{t}$ & $\begin{array}{l}\text { Costa-Rica (2), Honduras (2), } \\
\text { Equateur-Ecuador (2) }\end{array}$ \\
\hline Caoutchouc & Kautschuk & $2250000 \mathrm{t}$ & $\begin{array}{l}\text { Indonésie-Indonesien (15), } \\
\text { Malaisie-Malaya (12) }\end{array}$ \\
\hline Colza & Raps & $130000 \mathrm{t}$ & Suède-Schweden (1) \\
\hline $\begin{array}{l}\text { Tourteaux et } \\
\text { farine de t. }\end{array}$ & $\begin{array}{l}\text { Ölsaatkuchen } \\
\text { und -mehl }\end{array}$ & $2316600 \mathrm{t}$ & Argentine-Argentinien (1) \\
\hline Coprah & Kopra & $1513300 \mathrm{t}$ & $\begin{array}{l}\text { Philippines-Philippinen (7), } \\
\text { Indonésie-Indonesien (4) }\end{array}$ \\
\hline $\begin{array}{l}\text { Huile de coco } \\
\text { Palmistes }\end{array}$ & $\begin{array}{l}\text { Kokosöl } \\
\text { Palmkerne }\end{array}$ & $\begin{array}{l}370000 \mathrm{t} \\
768300 \mathrm{t}\end{array}$ & Nigeria-Nigerien (4) \\
\hline
\end{tabular}

* Les chiffres se rapportent au nombre des signes du pays en question

* Die Ziffern beziehen sich auf die Zahl der Signaturen dieses Landes 


\begin{tabular}{|c|c|c|c|}
\hline Produit & Produkt & $\begin{array}{c}\text { Total du commerce } \\
\text { mondial sans U.R.S.S. } \\
\text { Weltexportmenge } \\
\text { ohne U.S.S.R. } \\
\text { Moyenne - Mittel } \\
1951-1953\end{array}$ & $\begin{array}{l}\text { Principaux pays exportateurs } \\
\text { Wichtigste Exportländer }\end{array}$ \\
\hline $\begin{array}{l}\text { Huile de palme } \\
\text { Soya } \\
\text { Huile de soya } \\
\text { Arachides } \\
\text { Huile d'arachide } \\
\text { Huile d'olive } \\
\text { Huile de lin } \\
\text { Coton } \\
\text { Huile de coton } \\
\text { Jute } \\
\text { Sisal } \\
\text { Soie } \\
\text { Laine }\end{array}$ & $\begin{array}{l}\text { Palmöl } \\
\text { Sojabohnen } \\
\text { Sojaöl } \\
\text { Erdnüsse } \\
\text { ErdnuBöl } \\
\text { Olivenöl } \\
\text { Leinöl } \\
\text { Baumwolle } \\
\text { Baumwollsaatöl } \\
\text { Jute } \\
\text { Sisal } \\
\text { Seide } \\
\text { Wolle }\end{array}$ & $\begin{array}{r}523300 \mathrm{t} \\
1343300 \mathrm{t} \\
150000 \mathrm{t} \\
730000 \mathrm{t} \\
\\
213300 \mathrm{t} \\
98300 \mathrm{t} \\
\\
255300 \mathrm{t} \\
2410000 \mathrm{t} \\
77300 \mathrm{t} \\
986600 \mathrm{t} \\
+10000 \mathrm{t} \\
6300 \mathrm{t} \\
686600 \mathrm{t}\end{array}$ & $\begin{array}{l}\text { Nigeria-Nigerien (2) } \\
\text { E.U.-U.S.A. (3) } \\
\text { E.U.-U.S.A. (2) } \\
\text { Afrique-occ.franc.-Franz. West- } \\
\text { a frika (2), Nigeria-Nigerien (2) } \\
\text { Afrique-occ.franç.-Franz. West- } \\
\text { afrika (3) } \\
\text { Espagne-Spanien (1), Algérie-Al- } \\
\text { gerien (1), Tunisie-Tunis (1) } \\
\text { Argentine-Argentinien (2) } \\
\text { E.U.-U.S.A. (18), Egypte-Aegyp- } \\
\text { ten (6), Pakistan (5) } \\
\text { E. U.-U.S.A. (1) } \\
\text { Pakistan (10) } \\
\text { Tanganyika-Tanganjika (2) } \\
\text { Japon-Japan (4) } \\
\text { Australie-Australien (29), Nou- } \\
\text { velle-Zélande-Neuseeland (13), } \\
\text { Argentine-Argentinien (7) }\end{array}$ \\
\hline
\end{tabular}

\section{EINE NEUE KARTE DER WELTWIRTSCHAFT}

Die Karte der Weltwirtschaft vereinigt bildhafte Darstellung mit wissenschaftlicher Sachlichkeit. Den Hintergrund bilden die Hauptformen der Landnutzung (Flächenkolorit). Die Schwerpunkte der Weltwirtschaft sind die Bevölkerungs- und Industriezentren, welche, dank roten Signaturen, deutlich hervortreten. Der Bergbau (schwarz) ist mit seinen Produktionswerten dargestellt, während bei Land- und Forstwirtschaft (grün, blau usw.) die Exportwerte berücksichtigt wurden, um die weltwirtschaftliche Bedeutung der verschiedenen Länder zu akzentuieren. Die einzelnen Signaturen sind lagerichtig eingetragen und repräsentieren angenähert denselben Handelswert. Damit die wirtschaftliche Bedeutung eines Gebietes aus der Signaturenhäufung ersichtlich wird, mußte bei der graphischen Gestaltung der Signaturen auch der Intensitätswert (gegeben durch Form, Größe und Farbe) berücksichtigt werden. Die konsequente Anwendung der Einzelsignatur erlaubt eine eindeutige Interpretation des Karteninhaltes, die wirklichkeitsnahen Symbole erleichtern seine rasche Erfassung und beleben das Kartenbild. Der Begleittext enthält die ausführlichen Erklärungen zur Methodik und Legende; er orientiert auch über die Unterlagen.

\section{37. JAHRESVERSAMMLUNG DER SCHWEIZERISCHEN NATURFORSCHENDEN GESELLSCHAFT}

\section{RENÉ NERTZ}

Im Rahmen der 137. Jahresversammlung der Schweizerischen Naturforschenden Gesellschaft führte der Verband der Schweizerischen Geographischen Gesellschaften am 21./22. September 1957 in Neuenburg seine Jahrestagung durch. Im Naturkundezimmer des neu erbauten Gymnasiums stand ein für alle $Z$ wecke der Demonstration und Projektion besteingerichteter Raum zur Verfügung.

Der Präsident des Verbandes, Dr. ERICH SchwaBE, konnte an der vorausgehenden Delegiertenversammlung die Vertreter aller Gesellschaften begrüßen. Im Mittelpunkt dieser Geschäftssitzung stand der Bericht des Präsidenten der Forschungskommission, Prof. Dr. H. Gutersohn (ETH), über deren Tätigkeit. Seine Ausführun- 